\title{
La conceptualización errada del campo eléctrico con cálculo integral en clases virtuales en estudiantes de física aplicada
}

\author{
S. Coello Pisco ${ }^{a, b}$, J. Flores Herrera ${ }^{a}$, J. Hidalgo Crespo $^{a}$ y Y. González Cañizalez ${ }^{a}$ \\ ${ }^{a}$ Departamento de Investigación, Facultad de Ingeniería Industrial. Universidad \\ Guayaquil, Avenida 10 NO, Guayaquil, D.C Ecuador. \\ ${ }^{b}$ Departamento de Ingeniería Industrial. \\ ETSNM, campus Riazor. Universidad Da Coruña, Galicia, D.C. España.
}

Received 14 July 2021; accepted 13 September 2021

\begin{abstract}
En este trabajo se implementó un método basado en el teorema de Vergnaud sobre la conceptualización del campo eléctrico con cálculo integral realizado en una universidad privada de la ciudad de Guayaquil. El objetivo de este estudio es relacionar y conectar la interacción que existe entre el dominio conceptual-epistemológico (Saber comprender) con el hacer del dominio metodológico (Saber hacer) con el propósito de ampliar y profundizar el concepto mediante la experimentación a través de un material instruccional en las clases virtuales (Teoría de Gagné). Nuestro trabajo se fundamenta en la investigación acción con un enfoque explicativo, ya que desde la óptica de la práctica pedagógica pretendemos dar respuesta a los problemas de conceptualización sobre las variables que participan en el campo eléctrico. El diseño del estudio es experimental donde trabajó con grupos intactos, uno de control (Gc) y otro experimental (Ge). Los resultados indican que el grupo experimental desarrolló una mejor conceptualización en los contenidos de campo eléctrico con cálculo integral a diferencia del grupo de control que recibió una instrucción tradicional.
\end{abstract}

Descriptores: Campo eléctrico integral; dominio conceptual; dominio epistemológico; dominio metodológico; clases virtuales; teoría de Gagné; teoría de Vergnaud.

In this work, a method based on Vergnaud's theorem on the conceptualization of the electric field with integral calculus carried out in a private university in the city of Guayaquil was replicated. The objective of this study is to relate and connect the interaction that exists between the conceptual-epistemological domain (Knowing how to understand) with the doing of the methodological domain (Knowing how to do) in order to broaden and deepen the concept through experimentation through a material instructional in virtual classes (Gagné Theory). Our work is based on action research with an explanatory approach, since from the perspective of pedagogical practice we intend to respond to the problems of conceptualization about the variables that participate in the electric field. The study design is experimental, working with intact groups, a control group (Gc) and an experimental group (Ge). The results indicate that the experimental group developed a better conceptualization in the electric field contents with integral calculation, unlike the control group that received traditional instruction.

Keywords: Integral electric field; conceptual domain; epistemological domain; methodological domain; virtual classes; Gagné's theory; Vergnaud's theory.

DOI: https://doi.org/10.31349/RevMexFis.19.010203

\section{Introducción}

Los estudiantes que están aprendiendo Física presentan dificultad en la comprensión de conceptos en ciertos tópicos de la asignatura y debido al confinamiento dispuesto por las autoridades ecuatorianas por causa de la pandemia del virus SARSCoV-2 se debe impartir clases virtuales sincrónicas, lo cual es un evento no favorable para muchos estudiantes que no gozan de estas destrezas. Actualmente el avance tecnológico nos exige que los futuros egresados de las universidades utilicen la percepción espacial y la visualización de objetos en el espacio, que capten relaciones de capacidad para leer e interpretar representaciones bidimensionales y tridimensionales, desarrollar la habilidad del pensamiento y aplicar estrategias de resolución de problemas reales que se produzcan en sus labores seglares. El desarrollo tecnológico plantea que los futuros profesionales en ingeniería resuelvan en sus tra- bajos problemas ligados por una parte al cálculo de diversas estructuras estáticas y por otra, al diseño y construcción, explotación de todo tipo de motores y mecanismos industriales (Industria 4.0). En otras palabras, cómo ellos conceptualizan y de qué manera ponen en acción los conocimientos existentes en su estructura cognoscitiva. De acuerdo con lo antedicho, las ciencias físicas en el contexto educativo a través del tiempo han venido presentando inconvenientes en cuánto a la metodología o forma de explicarse las leyes básicas que rigen a los fenómenos que ocurren en la naturaleza. En su sentido más amplio, la Física utiliza herramientas que se fundamentan en las habilidades del pensamiento matemático y geométrico a través de las cuales se deducen propiedades y se formulan leyes que rigen a los fenómenos físicos; cómo podemos observarlos tanto en la naturaleza o los que se crean artificialmente en los laboratorios de investigación [1]. 
Según la teoría de Vergnaud, los campos conceptuales se basan en un conjunto heterogéneo e informal de situaciones problemáticas, conceptualizaciones, estructuras relacionadas, contenidos y operaciones de pensamiento que probablemente se enlazan en el proceso de adquisición de conocimientos [2]. Es decir, cada estudiante tiene un funcionamiento cognitivo diferente y por ende se pueden presentar dos contextos. El primero es que se puede dar un amplio repertorio de esquemas que le permita resolver adecuadamente el problema propuesto $[3,4]$. El segundo es todo lo contrario, al carecer de esquemas no podrá resolver la situación problemática propuesta de manera eficaz [5]. Otro escenario es la posibilidad de que el significado que el estudiante maneje sea un significado propio, es decir, que se relaciona con sus ideas previas. También existe la posibilidad de que el significado le sea irrelevante, al menos en la práctica, sino que pase directamente del enunciado a la representación matemática (desarrollo mecánico), en donde aplica a ciegas fórmulas y que es una actuación bastante habitual [6]. Para ejemplificar, los cursos del básico de la carrera de Ingeniería Industrial de la Universidad de Guayaquil (UG), en el primer y segundo semestre de estudio tiene en su malla curricular la asignatura de Física Aplicada y Cálculo diferencial e integral, en los cuáles se estudian conceptualizaciones que pueden guardar relación entre ambas disciplinas [7].

No obstante, ambas asignaturas requieren del dominio conceptual [8,9] para su correcto aprendizaje, aprehensión de contenidos y de situaciones problemáticas basadas en el campo de la ingeniería [10]; se debe recordar que los problemas pueden tener dos vías o están bien estructurados o mal estructurados. Según Carcavilla (2004), "un problema" sería una situación o conflicto en el cual no se tiene una respuesta inmediata ni algoritmo ni heurístico [11]. Es decir, no se sabe qué información necesitamos para intentar obtener una respuesta. Los problemas de resolución se caracterizan por ser: de nivel lingüístico, nivel conceptual y nivel físico [5]. El problema se resuelve en el nivel conceptual, con la necesaria intervención del nivel lingüístico y para ello se precisa hacer una representación del mismo en dichos niveles [1].

Para dar respuesta a este estudio sobre los problemas de conceptualización y ver si son correctos o no sus conceptos o teoremas, procedemos realizar un experimento desde la óptica del teorema de Vergnaud, replicado en los estudiantes de Física aplicada de la Facultad de Ingeniería Industrial (UG). Se busca hacer un estudio comparativo con un escenario futuro sobre si las incidencias que afectaron al grupo de estudio realizado por los investigadores Flores y Coello [12], en una universidad privada (UP) guardan relación con los estudiantes de una universidad pública (UG) con un número mayor de participantes.

Según los resultados que se obtengan con la metodología de Vergnaud, se procederá aplicar un tratamiento apropiado que nos permitirá determinar e identificar las dificultades que tienen los estudiantes de la carrera básica de ingeniería en el instante de resolver problemas de Física aplicando el cálcu- lo diferencial e integral. Proponemos replicar el método en el tópico de física: "los problemas de campo eléctrico" que aplican el cálculo diferencial e integral y que se encuentran propuestos en los libros de Física para ingeniería (Hewitt, Sears). Estos problemas son bien estructurados con algoritmos situados en un contexto específico. En estos problemas los estudiantes de pregrado deben estar en la capacidad de identificar los conceptos claves, algoritmos y seleccionar la secuencia idónea que presente la situación problemática del ejercicio a resolver. Cabe mencionar, que la enseñanza de la Ciencia comprende dos aspectos primordiales: "la conceptualización" y "la resolución del problema" y es a través de estos dos procesos que los conceptos se asimilan, y desarrol$\operatorname{lan}[13]$.

Finalmente, a partir de los resultados, nuestro objetivo consiste en relacionar y conectar la interacción que existe entre el dominio conceptual-epistemológico (Saber comprender) con el hacer del dominio metodológico (Saber hacer) con el propósito de ampliar y profundizar el concepto mediante la experimentación a través de un material instruccional en las clases virtuales. Por ende, fundamentamos este estudio a partir de las técnicas didácticas y modelos pedagógicos que permitan desarrollar las destrezas o habilidades cognitivas (pensamiento crítico) de los estudiantes en la asignatura de Física aplicada. Nos basamos en la teoría de Gagné cuyo enfoque está en el modelo de procesamiento de la información, el cual deriva de la posición semicognitiva de la línea tolmaniana [14-16]. A continuación exponemos la metodología a emplearse en el estudio y tratamiento a los participantes.

\section{Materiales y métodos}

El presente estudio tiene un enfoque de carácter explicativodescriptivo y se fundamenta en la investigación-acción. El diseño es experimental, la selección de la muestra es no probabilística incidental ya que se tiene acceso a ella y es representativa de la población [17]. Por otro lado, desde la óptica de la práctica pedagógica pretendemos dar respuesta a los problemas de conceptualización sobre las variables que participan en el campo eléctrico con herramientas de cálculo integral. En cuanto a la tarea instruccional fue un tema de la unidad de Campo eléctrico, contenido que fue previamente enseñado a los estudiantes y el tiempo empleado fue de seis horas $[18,19]$. El tiempo para la observación y entrevista fue de 40 minutos la cuál fue filmada y examinada para su respectivo análisis de las respuestas con respecto a los conceptos y teoremas en acción adquiridos por los estudiantes en el tema seleccionado [20]. Los participantes fueron 82 estudiantes, 12 mujeres y 70 hombres entre las edades de 18 a 23 años. A continuación exponemos el proceso para obtener la información respectiva y proponer un tratamiento para coadyuvar mejor la compresión conceptual de los estudiantes en las clases virtuales. 


\subsection{Fase 1: Identificación de los conceptos y teoremas de acción correctos e incorrectos}

Como siguiente fase, se identifican los conceptos y teorema de acción encontrado en los 82 participantes de la universidad pública (UG). El problema propuesto para esta fase consistía en determinar el campo eléctrico en un punto "P" a cierta distancia "n".

Problema propuesto: Se tiene una barra uniforme cuya longitud es $l$ la cual tiene una carga eléctrica por unidad de longitud $\lambda$. Construya un esquema gráfico que represente el problema propuesto y calcule el campo eléctrico en el punto "P" que se encuentra a lo largo del eje a una distancia "n" del extremo de la barra cargada eléctricamente. Codificación: Sistema de referencia (SR), diferencial de carga eléctrica $(\delta Q)$, diferencial de longitud $(\delta l)$, diferencial de campo eléctrico $(\delta E)$ y densidad lineal $(\lambda)[12,19]$.

\subsection{Fase 2. Aplicación del modelo de Gagné para mejo- rar la comprensión conceptual en la modalidad vir- tual}

En esta fase se trabaja con grupos intactos, uno de control $\left(\mathrm{G}_{c}\right)$ el cuál se trabajará de manera tradicional y otro experimental $\left(\mathrm{G}_{e}\right)$ el cuál se aplicará el plan de curso según Gagné. Se procede a preparar un diseño basado en los resultados obtenidos, se propone aplicar nueve pasos para elaborar un plan de clase síncrono que depende en gran medida de las condiciones internas (procesos) y externas (un aprendizaje óptimo). En las condiciones internas se producen nueve eventos instruccionales a seguir y en las externas son de cinco tipos de capacidad a desarrollar (Tabla I).

Además según las fases del aprendizaje de la teoría de Gagné se propone para mejorar la conceptualización del grupo experimental a través de los siguientes descriptores adjuntos en la Tabla II.

TABLA I. Descripción de las condiciones a aplicarse para el plan de curso en el tema de campo eléctrico.

\begin{tabular}{ll}
\hline Condiciones internas & Condiciones externas \\
\hline Ganar atención & Información verbal \\
Informar objetivos & (conocimiento) \\
Estimular el & Destrezas \\
conocimiento previo & intelectuales \\
Presentar material nuevo & (conocimiento \\
Guiar el aprendizaje & práctico) \\
Suscitar el rendimiento & Estrategias \\
individual & cognitivas \\
Proporcionar & (Plan de acción) \\
retroalimentación & Actitudes \\
Evaluar la eficacia & Destrezas motoras \\
del rendimiento & \\
Incrementar la retención & \\
\hline
\end{tabular}

TABLA II. Fases del aprendizaje y descriptores a utilizarse para mejorar el nivel conceptual del estudiantado.

\begin{tabular}{|c|c|c|}
\hline Fase & Aumentar & Disminuir \\
\hline $\begin{array}{l}\text { Aprendizaje } \\
\text { en cadena } \\
\text { Respuestas } \\
\text { operantes }\end{array}$ & $\begin{array}{l}\text { Uso de } \\
\text { materiales del } \\
\text { entorno del hogar } \\
\text { (Laboratorio de } \\
\text { Física doméstica) }\end{array}$ & $\begin{array}{l}\text { Practica } \\
\text { mecánica. } \\
\text { Memorizar } \\
\text { formulas } \\
\text { geométricas }\end{array}$ \\
\hline $\begin{array}{l}\text { Aprendizaje } \\
\text { de conceptos. } \\
\text { Aprendizaje } \\
\text { de principios. } \\
\text { Aprendizaje } \\
\text { de signos. } \\
\text { Asociaciones } \\
\text { verbales }\end{array}$ & $\begin{array}{l}\text { Discusiones } \\
\text { manteniendo un } \\
\text { lenguaje } \\
\text { matemático y } \\
\text { físico } \\
\text { identificando } \\
\text { mediante } \\
\text { analogías entre } \\
\text { una y otra } \\
\text { asignatura. }\end{array}$ & $\begin{array}{l}\text { Memorización } \\
\text { mecánica de } \\
\text { reglas y } \\
\text { formulas. } \\
\text { Respuestas } \\
\text { únicas y } \\
\text { métodos únicos } \\
\text { para encontrar } \\
\text { respuestas. }\end{array}$ \\
\hline $\begin{array}{l}\text { Aprendizaje de } \\
\text { discriminaciones } \\
\text { múltiples }\end{array}$ & $\begin{array}{l}\text { Cuestionar y } \\
\text { realizar } \\
\text { conjeturas. } \\
\text { Justificación del } \\
\text { pensamiento. } \\
\text { Escribir acerca } \\
\text { de la Física } \\
\text { y matemáticas. } \\
\text { Uso de } \\
\text { geometría en } \\
\text { solución de } \\
\text { problemas. } \\
\text { Trabajo } \\
\text { cooperativo- } \\
\text { colaborativo } \\
\text { vía zoom con } \\
\text { supervisión } \\
\text { del docente. } \\
\text { Desarrollar } \\
\text { habilidades de } \\
\text { construcción de } \\
\text { dibujos e } \\
\text { interpretación de } \\
\text { gráficas desde } \\
\text { el punto de } \\
\text { vista } \\
\text { matemático } \\
\text { al físico. }\end{array}$ & $\begin{array}{l}\text { Uso de } \\
\text { hojas de } \\
\text { ejercicios } \\
\text { rutinarios. } \\
\text { Practicas } \\
\text { escritas } \\
\text { repetitivas. }\end{array}$ \\
\hline
\end{tabular}

\section{Supuestos:}

Ho: La aplicación de la Teoría de Gagné no incide de manera significativa en los conceptos y teoremas de acción incorrectos de los estudiantes de Física aplicada.

Ha: La aplicación de la Teoría de Gagné incide de manera significativa los conceptos y teoremas de acción incorrectos de los estudiantes de Física aplicada. 
TABLE III. Conceptos y Teoremas en acción incorrectos de ambos grupos GE y GC

\begin{tabular}{|c|c|c|}
\hline Dimensión & $\begin{array}{l}\text { Conceptos } \\
\text { en acción }\end{array}$ & $\begin{array}{l}\text { Teoremas en } \\
\text { acción }\end{array}$ \\
\hline 1 & $\begin{array}{l}\text { Interpretación } \\
\text { de gráfica }(S R)\end{array}$ & $\begin{array}{l}\text { El SR se localiza } \\
\text { en cualquier punto } \\
\text { El SR no necesita de } \\
\text { ubicación y/o selección }\end{array}$ \\
\hline 2 & $\begin{array}{l}\text { Diferencial de carga } \\
\text { eléctrica }(\delta Q) \\
\text { Diferencial de } \\
\text { longitud }(\delta l)\end{array}$ & $\begin{array}{l}\text { El } \delta l \text { produce un } \delta E \\
\text { El } \delta l \text { requiere de selección } \\
\text { El } \delta l \text { tiene un espesor finito }\end{array}$ \\
\hline 3 & $\begin{array}{l}\text { Distancia del } \\
\text { SR hasta } \delta Q\end{array}$ & $\begin{array}{l}\text { No requiere } \\
\text { de determinación }\end{array}$ \\
\hline 4 & $\begin{array}{l}\text { Diferencial de } \\
\text { campo eléctrico } \\
(\delta E)\end{array}$ & $\begin{array}{l}\delta E \text { produce un } \\
\text { campo total } \delta E \\
\text { no requiere } \\
\text { representación } \\
\text { El segundo miembro } \\
\text { de la expresión } \\
\text { de campo eléctrico } \\
\text { es un escalar }\end{array}$ \\
\hline \multirow[t]{3}{*}{5} & $\begin{array}{l}\text { Distancia de } \delta Q \\
\text { hasta el punto "P" }\end{array}$ & $\begin{array}{l}\text { No hay respuesta de } \\
\text { la distancia } \delta E \\
\text { hasta el punto "P" }\end{array}$ \\
\hline & $\begin{array}{l}\lambda \text { de } \\
\text { carga eléctrica }\end{array}$ & $\begin{array}{l}\text { La densidad de carga } \lambda \\
\text { es definida como el } \\
\text { producto entre la carga } \\
\text { por unidad de longitud }\end{array}$ \\
\hline & $\begin{array}{l}\text { Límites de } \\
\text { Integración }\end{array}$ & $\begin{array}{l}\text { Los límites de integración } \\
\text { abarca todo el } \\
\text { sistema de carga }\end{array}$ \\
\hline
\end{tabular}

\section{Resultados y discusión}

El análisis del proceso de resolución de problemas reveló las siguientes situaciones (Tabla III) que se detallan a continuación con respecto a los conceptos y teoremas de acción por parte de los participantes en este estudio de la UG.

\subsection{Dimensión 1 conceptos y teoremas en acción según la teoría de Vergnaud: Interpretación de gráfica}

Al aplicar la metodología propuesta sobre la teoría de Vergnaud a ambos grupos se obtuvo que los estudiantes presentan problemas de reconocimiento e interpretación de gráficas, esto se debe al instante de conceptualizar el término "campo eléctrico" tanto el grupo de control y experimental muestras un alto porcentaje en identificar los límites del problema propuesto con una mínima diferencia del $2 \%$, ubicación y distancia al punto "P" oscilan entre $86.6 \%$ a un $91.5 \%$ y en cuánto a identificación de parámetros entre $87.8 \%$ a $89.4 \%$ (Figs. 1 y 2).

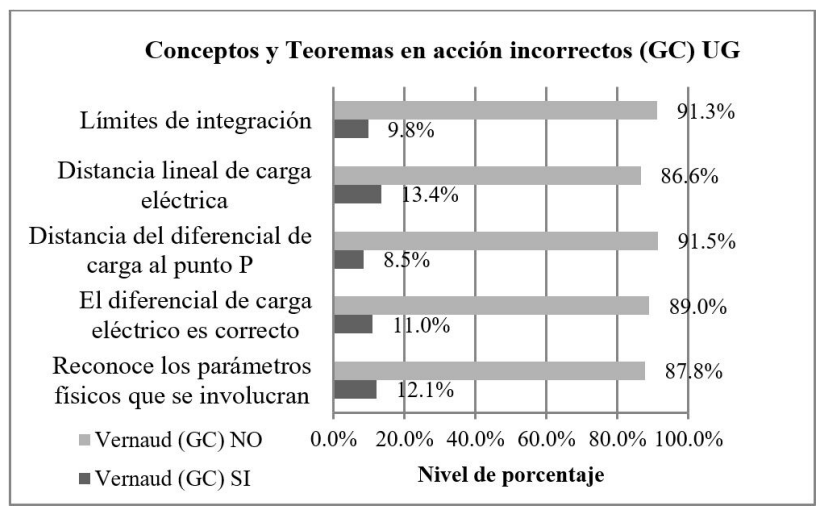

FIGURA 1. Se muestra los niveles de porcentaje obtenido de los conceptos y teoremas de acción incorrectos del grupo de control antes.

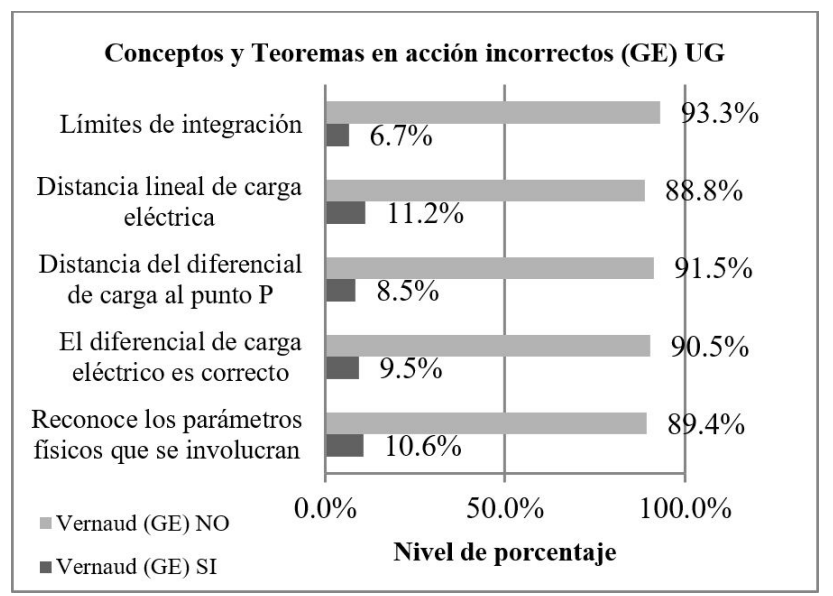

FIGURA 2. Se muestra los niveles de porcentaje obtenido de los conceptos y teoremas de acción incorrectos del grupo experimental antes de ser aplicado el tratamiento para mejorar la conceptualización.

A ambos grupos se les aplicó la teoría de los campos conceptuales de Vergnaud para identificar si sus conceptos y teoremas en acción estaban correcto o en qué nivel porcentual de error se encontraban (Fig. 1 y 2).

A la luz de los resultados se tiene que el $87.5 \%$ no reconoce los parámetros físicos que involucran al problema y no lograron ubicarse correctamente en el sistema de referencia (SR) entre un valor que oscila entre el 86.6-91.5\%. Estos son los datos reflejados en los estudiantes de Física aplicada de la UG (Fig. 3).

Basado en los resultados de Flores y Coello [12] mencionan que en la entrevista realizada a sus participante entrevistados, indican que un estudiante colocó el SR en el extremo derecho de la línea de carga eléctrica mientras que los participantes 2, 3 y 5 no hicieron la representación gráfica y el sexto estudiante lo colocó en el extremo izquierdo de la carga eléctrica, posterior a la resolución del problema. Una apreciación del estudiante " 1 " es que considera que: "en cálculo el sistema de referencia ya está dado o la gráfica ya está construida mientras que en física toca elaborarla”. En la Fig. 1 


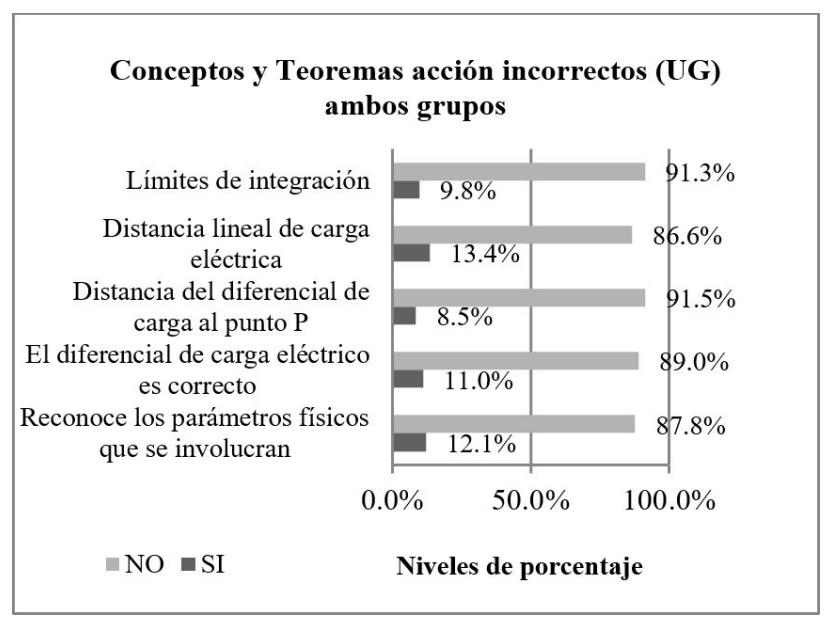

FIGURE 3. Se muestra los niveles de porcentaje obtenidos de los conceptos y teoremas de acción incorrectos de ambos grupos control y experimental antes de ser aplicado el tratamiento para mejorar la conceptualización.

y 2 con un escenario actual se puede apreciar que los estudiantes de la UG, no tiene un esquema definido para ubicarse en el espacio geométrico-abstracto del problema, mientras que los otros participantes no tienen esquemas para representar la posición del sistema de referencia, fenómeno replicado en ambas instituciones con tiempos muy distantes.

Teorema en acción: El razonamiento espacial-visualabstracto, es una base que junto a las habilidades espacialesgeométricas permiten al estudiante la asimilación de ejercicios e interpretación de gráficos en el aprendizaje de la Física. En otras palabras, la interpretación correcta del gráfico facilita a los estudiantes reconocer los parámetros físicos que se involucran en el problema propuesto. Esto permite establecer un bosquejo eficiente para aplicar una correcta estrategia y así poder iniciar la resolución del ejercicio propuesto [21]. Si el estudiante no construye la gráfica no podrá hacer un esquema que le permita interpretar el problema dado y resolverlo.

\subsection{Dimensión 2 concepto en acción: Diferencial de car- ga eléctrica $(\delta Q)$, distancia del SR hasta $\delta Q$, difer- encial de campo eléctrico $(\delta E)$ y definición de den- sidad eléctrica lineal $(\lambda)$}

El $89 \%$ no logró identificar el $\delta Q$ y trabajó con un $\delta l$. En cuanto a la distancia lineal de la carga al punto "P", el $91.5 \%$ no logra identificar las variables a trabajar como consecuencia de una mala interpretación del problema al construir la gráfica (Figs. 1 al 3). En esta fase los estudiantes seleccionados tenemos que el indicaron que $\delta l$ produce un $\delta E$, mientras que otros acotaron que el espesor del $\delta Q$ poseer el mismo orden de magnitud de las medidas relevantes del ejercicio. El resto de los estudiantes mencionaron que se debe seleccionar un $\delta l$. En el avance del proceso del problema tres estudiantes no representaron la distancia que hay desde el SR hasta el $\delta Q$. En cuanto a al $\delta E$ el $86.5 \%$ de los participantes omitieron la gráfica y el símbolo del $\delta E$ en el punto solicitado. Sólo un estudiante no representó el campo eléctrico en el punto solic- itado. En el penúltimo concepto de acción incorrecto sobre la distancia $\delta Q$ del hasta el punto "P" siete estudiantes expresaron la forma $(r+b)$ y durante la fase matemática procedimental de integración la consideraron como una constante. Otros estudiantes la formularon como $x$ pero luego corrigen y la expresa como: $(L+b-x)$, el $13.4 \%$ como $(l+b-x)$ y el resto lo expresa matemáticamente como $(l+b-x+d x)$. La densidad de carga eléctrica se define como un producto entre la carga eléctrica $(Q)$ y la longitud $(l)$.

Teorema en acción: "El produce un $\delta E ; \delta l$ es que requiere selección, $\delta l$ tiene un espesor finito". "Él $\delta E$ causa un campo total; $\delta E$ no precisa representación; el segundo miembro del campo eléctrico es un escalar"; "La distancia del $\delta E$ hasta el punto "P" no queda determinada y por ende no hay respuesta clara en esta parte procedimental". En el problema es necesario aclarar lo que se selecciona es un $\delta Q$ y no un $\delta l$; el primero tiene un espesor $\delta x$ y por ser una cantidad infinitesimal su espesor tiende a cero. Para calcular el área bajo la curva (esquema), es cierto que a una distancia $x$ del SR se toma valores de $\delta x$ y altura $y$; pero los estudiantes no lo trabajan desde el punto de vista de la Física sino más bien desde el punto de vista del cálculo diferencial lo que trae confusión al resolver el problema. El concepto de distancia del SR hasta el $\delta Q$ es importante, ya que de aquí se parte con la definición de la variable con respecto a la cual se introduce al proceso de integración (cálculo integral) los estudiantes tienden a confundir los procesos matemáticos con el procedimental físico ya que están familiarizados solo con las variables $x, y$ en los ejercicios de cálculo diferencial.

Por otra parte, es correcto que en el punto solicitado exista un campo eléctrico total $E$ pero se tiene que un $\delta Q$ en ese punto produce un $\delta E$ y se debe mantener la desigualdad vectorial; por lo que el segundo miembro debe expresarse de manera vectorial y no escalar como indicó un participante. Frente a los resultados obtenidos tanto de los 82 estudiantes como de los 6 participantes seleccionados por los investigadores Flores y Coello [12], procedemos a exponer que si los estudiantes no tienen desarrolladas ciertas habilidades específicas, no podrán avanzar de un nivel a otro sin poseer el nivel de razonamiento o conceptualización correcta que implican dichas habilidades. Con esto queremos explicar que: si un estudiante llega a un nivel de razonamiento en un contenido geométrico o matemático no asegura que frente a otro contenido nuevo para él pueda funcionar con el mismo nivel y tenga que recurrir a formas de razonamiento de los niveles anteriores según el orden de complejidad creciente [22,23]. Estos resultados nos muestran que a pesar de ser estudios en diferentes tiempos los problemas de conceptualización se encuentran en los diferentes niveles de educación y estrato social.

\subsection{Dimensión 3 concepto en acción: Límites de inte- gración}

Con respecto a la resolución del problema el $91.3 \%$ no logra resolver correctamente el ejercicio, sólo un $9.8 \%$ escribe 
correctamente los límites de integración pero sólo el $6.1 \%$ da una respuesta correcta al problema planteado (Fig. 3). Algunos estudios manifiestan este tipo de situación en los estudiantes de carrera de pregrado $[11,24]$. Los límites de integración parten de un buen SR circunscribiéndose a la longitud de la línea de carga eléctrica. En este estudio se obtuvo un total de 8 estudiantes que procedieron correctamente en aplicar los pasos procedimentales para resolver el ejercicio propuesto hasta los límites de integración pero sólo 5 estudiantes obtuvieron el resultado correcto de los 84 estudiantes mientras que en el estudio de Flores y Coello sólo uno del grupo de entrevistado dio con la respuesta [12]. Estos resultados nos indican que muchos estudiantes son capaces de dar solución a una problemática pero que ello no implica que tenga una buena comprensión conceptual $[25,26]$. En otras palabras, los estudiantes en este nivel de pregrado deben aprender a diferenciar los procesos y reconocer sus principales características para poder expresar de forma matemática. El resultado es que al no relacionar matemáticamente los parámetros físicos unos con otros, los induce a pensar que no hay relación alguna por ende sus concepciones alternas son equivocadas. Esto se debe a que se enfocan en cómo usar el cálculo diferencial e integral en el problema. Su concentración está en la aplicación, y no en la comprensión del fenómeno físico que éste con lleva cuando se resuelven problemas de física conduciéndolos al pensamiento equivocado. Esto nos demuestra que tener presentes los conceptos y teoremas en acción da excelentes resultados y permite al estudiante una comprensión conceptual adecuada. Las concepciones y teoremas en acción que precisan ser mejoradas son: Construcción de gráficas, interpretación del gráfico, conceptualización del campo eléctrico, densidad de carga, límites de integración.

\subsubsection{Aplicación del tratamiento según Gagné}

A la luz de las deducciones observadas, aplicamos un tratamiento didáctico con fundamentación en la Teoría de

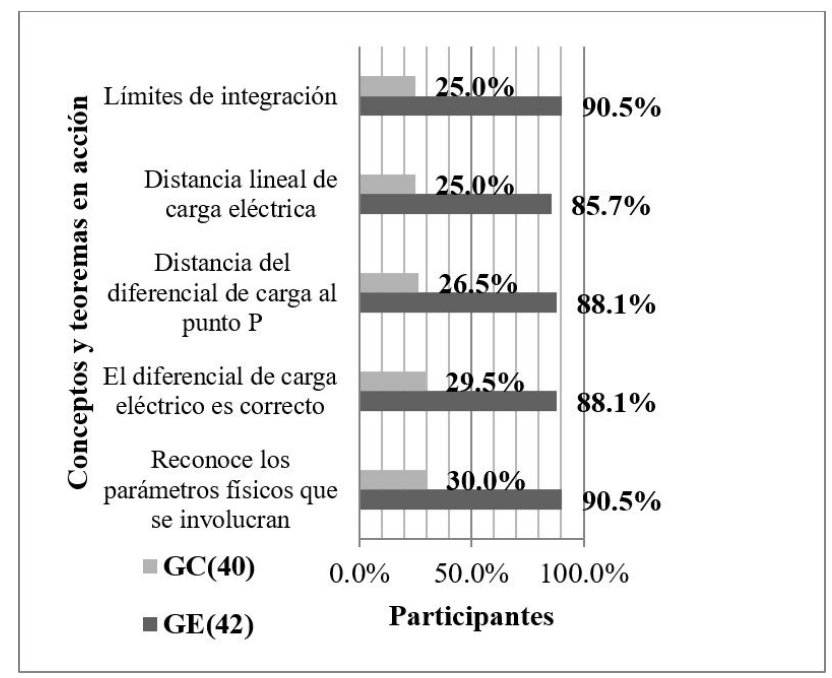

FIGURE 4.Resultados de aplicar el tratamiento al grupo experimental según la teoría de Gagné para el curso de Física Aplicada.

\begin{tabular}{lll}
\hline \multicolumn{2}{l}{ TABLA IV. Prueba F para varianzas de dos muestras. } \\
\hline & GE & GC \\
\hline Media & 8.714484127 & 6.053461538 \\
Varianza & 1.41505779 & 5.910505466 \\
Observaciones & 42 & 39 \\
Grados de libertad & 41 & 38 \\
$\mathrm{~F}$ & 0.239414006 & \\
$\mathrm{P}(\mathrm{F}<=$ f) una cola & $7.44243 \mathrm{E}-06$ & \\
Valor crítico & & \\
para F (una cola) & 0.590315801 &
\end{tabular}

*Nivel de significancia, $p<0,0.5$.

TABLA V. Prueba $t$ para dos muestras suponiendo varianzas desiguales.

\begin{tabular}{lll}
\hline & GE & GC \\
\hline Media & 8.714484127 & 6.11475 \\
Varianza & 1.41505779 & 5.909205064 \\
Observaciones & 42 & 40 \\
Diferencia hipotética & & \\
de las medias & 0 & \\
Grados de libertad & 56 & \\
Estadístico $t$ & 6.103570784 & \\
$\mathrm{P}(\mathrm{T}<=\mathrm{t})$ una cola & $5.15383 \mathrm{E}-08$ & \\
Valor crítico & & \\
de $t$ (una cola) & 1.672522303 & \\
$\mathrm{P}(\mathrm{T}<=t)$ dos colas & $1.03077 \mathrm{E}-07$ & \\
Valor crítico de & & \\
$t($ dos colas) & 2.003240719 & \\
\hline
\end{tabular}

*Nivel de significancia, $p<0,0.5$.

Gagné (Tabla I y II) para lo cual obtuvimos los siguientes resultados:

El grupo experimental mejoró de manera significativa sus conceptualizaciones y teoremas de acción incorrectos con un porcentaje que oscila entre $88.1 \%$ a $90.5 \%$. Mientras el grupo de control se impartió la clase tradicional vía virtual de tal manera que sus índices de mejoramiento alcanzan apenas entre un $30 \%$ con un $25 \%$ del estudiantado que llega a la respuesta (Fig. 4). Se procedió a realizar la prueba Fisher al grupo experimental y de control en los cuales se obtuvo que las diferencias de las medias y varianzas sean significativas (Tabla IV).

$\mathrm{F}_{\text {calculada }}<\mathrm{F}_{\text {crítico }}$ se acepta que existe alguna diferencia en la variación de los resultados obtenidos del tratamiento aplicado en el grupo experimental vs el grupo de control aplicando la Teoría de Gagné en el curso de Física Aplicada (Tabla IV).

A la luz de los resultados obtenidos en la Tabla Vtenemos que: $t_{\text {empírico }}>t_{\text {crítico }}$ se rechaza Ho; como consecuencia se acepta Ha; $6.1036>1.6725$.

Decisión estadística: La aplicación de la Teoría de Gagné puede afectar de manera significativa los conceptos 
y teoremas de acción incorrectos de los estudiantes de Física aplicada.

\section{Conclusiones}

En este estudio hemos demostrado como las percepciones alternas inciden en la resolución de problemas físicos relacionados con el cálculo diferencial e integral en estudiantes de pregrado de la carrera de ingeniería industrial con un escenario futuro y replicando un trabajo realizado en un tiempo distinto al actual. Al introducir la teoría de campos conceptuales se lograron identificar los conceptos y teoremas en acción errados en un tópico del campo de la enseñanza de la Física. Dicho estudio puede ser replicado en cualquier otra asignatura en los diferentes niveles educativos. El siguiente paso fue diseñar un material instruccional didáctico en donde se implementó los resultados de este trabajo y aplicando el tratamiento adecuado se desarrollaron las condiciones internas, externas para mejorar las habilidades de pensamiento crítico de los estudiantes (conceptos y teoremas en acción) en el curso de Física Aplicada.

Por ende, recomendamos que la función del profesor no se limite a la transmisión de conocimientos sino que, además, estimule en los estudiantes el propio deseo de adquirir conocimientos y despertar su espíritu crítico, es decir, el estudiante es el centro del aprendizaje y el fin es obtener una buena calidad de la enseñanza como se demostró en este estudio a través de metodologías y técnicas didácticas para mejorar las destrezas y competencias de los estudiantes.
1. M. A. Bunge, El planteamiento CientÃfico, Rev. Cub. Salud Pública 43 (2017) 470.

2. M. A. Moreira, La Teoría de los campos conceptuales de Vergnaud, la enseñanza de las ciencias y la investigación en el área, Inv. Ens. Cienc. 7 (2002) 7, https://www.if.ufrgs.br/cref/ojs/index. php/ienci/article/view/569/361

3. D. H. Jonassen, Toward a Design Theory of Problem Solving, Educ. Technol. Res. Dev. 48 (2000) 63, https : //www. jstor.org/stable/30220285

4. F. J. Quiroga Villegas y G. B. Andino, Problemas bien y mal estructurados en la evaluación diagnóstica de competencia en aspirantes a ingresar a carreras de ingeniería, http://eduqa2013.eduqa. net/eduqa2013/images/ponencias/eje2/ 2-9-Quiroga_Andino_Problemas_bien_y_mal. estructurados_en_la_evaluacion_diagnostica. de_competencia_en_aspirantes_a_ingresar_a. carreras_de_Ingenieria.pdf

5. M. R. Otero, M. A. Fanaro, P. Sureda, V. Carolina y M. Arlego, La Teoría de los Campos Conceptuales y la conceptualización en el aula de Matemática y Física (Dunken, Buenos Aires, 2014).

6. M. Molina, Métodos de resolución de problemas (Fundación General de la Universidad Politécnica de Madrid, Madrid, 2006), 4ta ed., https://oa.upm.es/14207/1/ 06-metodos-resolucion-problemas.pdf

7. P. Camarena Gallardo, La matemática en el contexto de las ciencias, Innov. Educ. 9 (2009) 15, https://www. redalyc.org/articulo.oa?id=179414894003

8. A. Llancaqueo, El aprendizaje del concepto de campo en física: una investigación exploratoria a luz de la teoría de Vergnaud, Rev. Bras. Ens. Fis. 25 (2003) 399 https : / / www . redalyc.org/articulo.oa?id=179414894003

9. J. A. Acuña Díaz y D. C. Álvarez Saavedra, Aula virtual como herramienta didáctica en el proceso enseñanzaaprendizaje con el uso del paquete de ofimática, Rev. Educ.
Investig. 2 (2020) 66, https://doi.org/10.33996/ alternancia.v2i3.318

10. G. Mejia Aguilar y D. M. Franco Durán, Influencia de los dominios conceptuales en las competencias académicas: Área de física para ingeniería, Rev. Educ. Ing. 11 (2016) 32.

11. A. Carcavilla Castro y T. Escudero Escorza, Los conceptos en la resolución de problemas de física "bien estructurados": aspectos identificativos y aspectos formales, Ens. Cienc. 22 (2004) 213, https: //www7.uc.cl/sw_educ/educacion/grecia/ plano/html/pdfs/linea_investigacion/ Resolucion_de_Problemas_IRP/IRP_075.pdf

12. S. Coello Pisco y J. R. Flores Herrera, Dificultades en la aplicación del cálculo diferencial e integral en la resolución de problemas de campo eléctrico, Rev. Cient. YACHANA 2 (2013) 37, http://revistas.ulvr.edu.ec/index. php/yachana/article/view/38/33

13. M. Delgado, Modelo para la construcción de conceptos científicos en física, desde la teoría de los campos conceptuales Tesis doctoral. Universidad del Zulia, 2014.

14. M. A. Hernández Cano y A. A. Benítez Pérez, La enseñanza de las ciencias experimentales a partir del conocimiento pedagógico de contenido, Innov. Educ. 18 (2018) 141, https://dialnet.unirioja.es/servlet/ articulo?codigo $=6791105$

15. E. Gottberg de Noguera, G. Noguera Altuve y M. A. Noguera Gottberg, El aprendizaje visto desde la perspectiva ecléctica de Robert Gagné y el uso de las nuevas tecnologías en educación superior, Universidades 53 (2012) 50, https : / / www . redalyc.org/articulo.oa?id=37331092005

16. N. Olmedo Torre y O. Farrerons Vidal, Modelos Constructivistas de Aprendizaje en Programas de Formación (OmniaScience, Terrasa, 2017), https://doi.org/10.3926/ oms.367

17. P. López Roldan y S. Fachelli, Metodología de la investigación social cuantitativa. (Universitat Autónoma de Barcelona, Barcelona, 2015), 1era ed. 
18. P. Hewitt, Física conceptual. (Pearson Educación, México 2004).

19. F. Sears, M. Zemansky, H. Young y R. Freedman, Física universitaria, 11a Ed. (Pearson Education, California, 2006).

20. M. M. Andrés y A. M. Pesa, Conceptos-en-acción y teoremas-en-acción en un trabajo de laboratorio de física, Rev. Bras. Pesqui. Educa. Cienc. 4 (2004) 59, https://periodicos.ufmg.br/index.php/ rbpec/article/view/4096

21. J. L. González Marí, Resolución de problemas de matemáticas, diferentes clases y métodos de resolución, planificación, gestión de los recursos, representación, interpretación y valoración de los resultados. Estrategias de intervención educativa, https:// www.yumpu.com/es/document/read/22041792/ resolucion-de-problemas-dematematicas

22. M. Hegarty, Spatial Thinking in Undergraduate Science Education, Spat. Cogn. Comput. 14 (2014) 142, https: / / doi. org/10.1080/13875868.2014.889696
23. S. M. Coello Pisco, J. R. Flores Herrera y J. Venegas Gallo, Diseño e implementación de una propuesta metodológica para la resolución de problemas en la interpretación de gráficos en el movimiento unidimensional, utilizando el aprendizaje autorregulado y colaborativo, Lat. Am. J. Phys. Educ. 10 (2016) 4318. htt: / / www . la jpe.org/dec16/4318. Silvia_2016.pdf

24. L. Cui, N. Sanjay Rebello y A. G. Bennett, College Students' Transfer from Calculus to Physics, AIP Conf. Proc. 818 (2006) 37, https://doi.org/10.1063/1.2177017

25. E. Castro Martínez, Resolución de problemas: ideas, tendencias e influencias en España, Invest. Educ. Mat. 12 (2008) 113, https://www.seiem.es/publicaciones/ archivospublicaciones/actas/Actas12SEIEM/ Seminario2Castro.pdf

26. M. B. Nakhleh, Are Our Students Conceptual Thinkers or Algorithmic Problem Solvers? Identifying Conceptual Students in General Chemistry, J. Chem. Educ. 70 (1993) 52, https : //doi.org/10.1021/ed070p52 